\title{
Analysis of Augmented Gain EDFA Systems using Single and Multi-wavelength Sources
}

\author{
Jyoti Gujral \\ Assistant Professor \\ Indo Global College of Engineering, Abhipur \\ (Mohali), Punjab, India
}

\author{
Vishu Goel \\ Student \\ S.D.D. I.E.T, Barwala (Panchkula), Haryana, India
}

\begin{abstract}
The scope of this paper is to analyze the performance of augmented gain EDFA systems using Single and Multiwavelength Input sources. The Performance of an Optical Communication system can be improved by the use of EDFAs as an Optical Amplifier. Erbium doped fiber amplifier (EDFA) is an important element in DWDM networks. We can achieve a flat gain spectrum by modeling the dynamic characteristics of an EDFA. This paper presents basic EDFA model operating on single (1550nm) and multi (1520-1610) $\mathrm{nm}$ wavelength operation with their simulation results. For the present work, we have used EDFA design software tool. It simulates various characteristics such as amplified spontaneous emission, gain, noise figure etc. in efficient manner. The working principle of this software provides accurate simulations and results. It confirms the excellent agreement between simulations and results obtained in real EDFA design. The proposed model consists of an input source, isolator, pump source, erbium fiber and WDM coupler. By changing the design parameters such as Input signal power and Pump Power, the different performance parameters (gain and noise figure) can be optimized. Without changing the values of isolator, erbium fiber length and WDM coupler and keeping the values of Input signal power $-30 \mathrm{dBm}$ and Pump Power $80 \mathrm{~mW}$ the obtained optimized gain is $34.45 \mathrm{~dB}$ for single wavelength source and $32.924 \mathrm{~dB}$ for multi wavelength source.
\end{abstract}

\section{General Terms}

Spectrum, Networks, Multiplexing.

\section{Keywords}

EDFA, Pumping, Isolator, WDM, ASE, Optical Fiber Communications, Augmented

\section{INTRODUCTION}

Now a days, EDFAs have been an interesting and active research area in the field of Optical Fiber Communication Systems [1].EDFAs are reliable for transmitting data through Long distance because of their wide bandwidth and optimum bit error rate. The light signal passing through the optical fiber is attenuated due to fiber absorption and scattering losses. To avoid this problem, One of the most efficient and versatile optical amplifier is EDFA. The use of EDFA as a Booster and Pre-amplifier [2] in an OFCS have enhanced the capacity of light wave systems beyond 1Tb/s.Fig.1 is showing an optical fiber system using EDFA as a booster and Pre- amplifier. EDFAs deployed in WDM networks provide Multi channel amplification with no cross talks.WDM technology [3] employing EDFAs will play a dominant role for next generation high speed networks. The ability [4] to pump the devices at different wavelengths and low coupling losses are the main features of an EDFA.EDFAs used together [8] with the Raman amplifiers provide ultra-low NF combined amplifiers which are used in very high bit rate links. The main advantages of EDFA are [9] high gain and low noise figure. The performance of EDFA is highly affected by Concentration of Erbium ions and temperature.

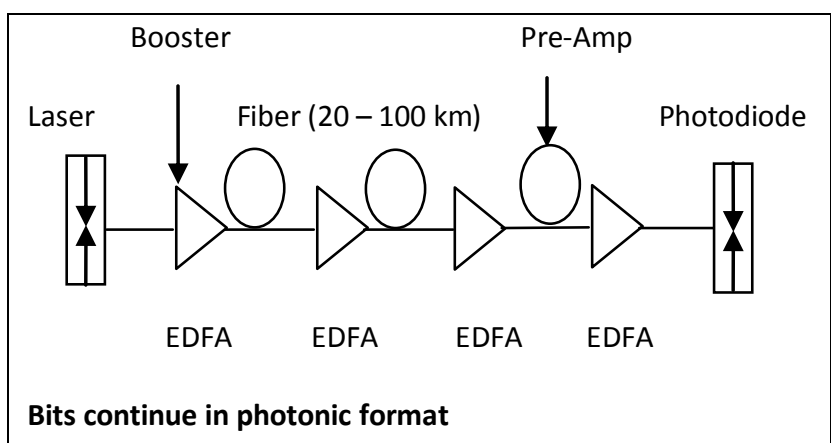

\subsection{Basic principle of EDFA}

The basic principle [10] for Amplification in an Erbium doped fiber amplifier is stimulated emission. In stimulated emission process, the light wave signal itself acts a stimulator for the emission of light. The doping agent [14] used in EDFA for Silica fiber core is trivalent erbium ions. Erbium provides minimum attenuation because it possesses an active transition at $1550 \mathrm{~nm}$ wavelength.

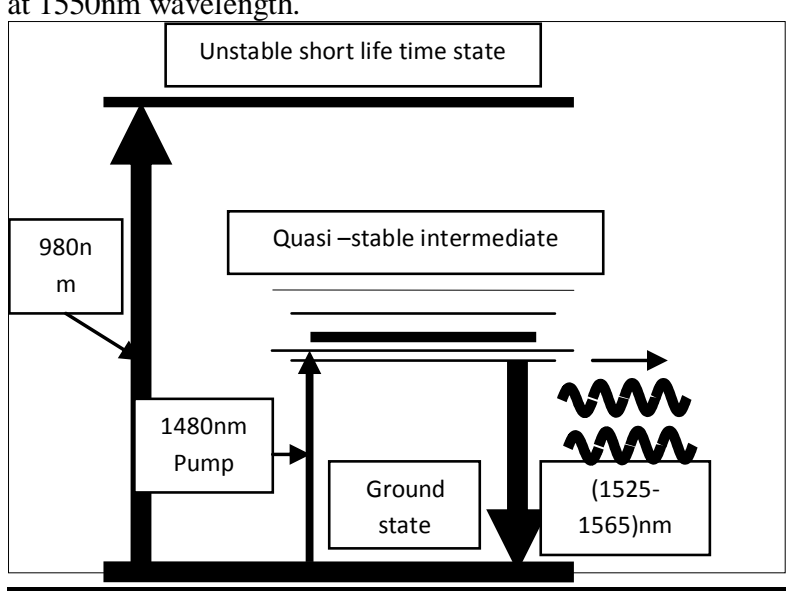

Fig.2: Physical Principle of EDFA 
Wavelength Selective Coupler [10] is used to mix a highpowered beam of light of (either $980 \mathrm{~nm}$ or $1480 \mathrm{~nm}$ ) from pumping source is mixed with the input signal. The mixed light is guided into a section of fiber with erbium ions included in the core. The Erbium ions are excited due to pumping source to a long lifetime intermediate state [13] as shown in Fig 2. When the photons belonging to the signal (at a different wavelength from the pump source) hit the excited erbium ions, the erbium ions give up some of their energy to the signal and return to their lower-energy state. A very important point is that erbium ions give up their energy in the form of photons of exactly same phase and direction as the input signal being amplified. Thus we can say, energy transfer will occur via the Erbium ions from the pump wavelength to the signal wavelength resulting in signal amplification[27] EDFAs can be designed to operate in forward -pumping configuration as well as backward -pumping configuration. In the forward pumping [5] configuration the input signal and the pump signal co-propagate with each other, while in the backward pumping they counter-propagate with each other. The gain and noise of an EDFA are greatly influenced by pump direction [15] because the pump direction changes the gain dynamics of amplifier. Pumping can be done at $660 \mathrm{~nm}$, $820 \mathrm{~nm}$ [30], 980nm and $1480 \mathrm{~nm}$ etc. The most widely used wavelength are $980 \mathrm{~nm}$ and $1480 \mathrm{~nm}$ [20]. Choi Bo - Hun et al [20] proposed a new pump wavelength of $1540 \mathrm{~nm}$ band for LBand EDFA by cascading both $980 \mathrm{~nm}$ and $1480 \mathrm{~nm}$ pump wavelengths. When EDFAs are used in cascading structure, the two major issues must be considered for good performance [6].First issue is Non-linearity of amplifier gain and second is noise introduced by the amplifier.Fig. 3 is showing the basic model consist of an input source, Pump source, WDM coupler and EDFA. Isolators [14] located before and after EDFA prevent pump signal from being transmitted along with the amplified signal.

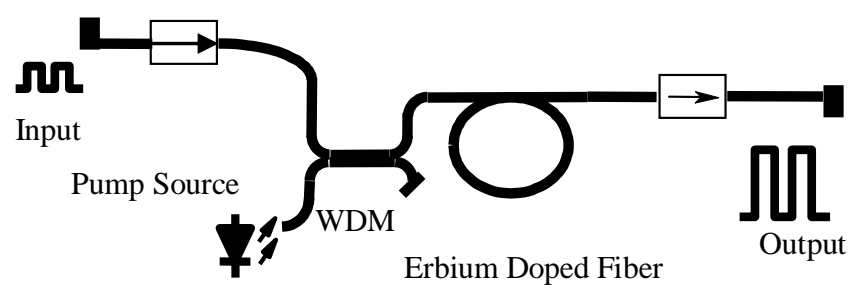

Fig 3: Basic EDFA model with all components

The principal source of noise in EDFAs is Amplified Spontaneous Emission (ASE) [3].It has a spectrum approximately the same as the gain spectrum [12] of the amplifier. The gain spectrum can be made extremely flat by optimizing the wavelength and pump power. Greater is the spontaneous emission, the greater will be the amplified spontaneous emission (ASE). As the nature of spontaneous emission [27] is incoherent, the amplified signal is noisier than the input signal. Kobayashi.M et al [29] proposed a new configuration for improving the noise figure of optical gain clamped fiber amplifier by mid-point band reject filter for lasing light. Erbium randomly emits photons between 1520 and $1570 \mathrm{~nm}$. Spontaneous emission (SE) is not polarized or coherent and like any photon, SE stimulates emission of other photons. When there is no input signal, eventually all optical energy is consumed into amplified spontaneous emission
(ASE) and contributes as a major source of noise in EDFA. Greater is the spontaneous emission, the greater will be the amplified spontaneous emission (ASE).

\subsection{Gain and Noise Figure in an EDFA}

The most important feature of the EDFA is gain. The gain of EDFA can vary from amplifier to amplifier. It can vary over a wide range depending on the amplifier length [27]. The shape of gain spectrum is affected, considerably by the nature of erbium ions. When a WDM signal is amplified .Gain is the ratio of output to the input light power and is given by [28]

Gain $=$ Pout $/$ Pin

Where (Pout) is the output power and (Pin) is the input power. A fast increase in the value of gain occurs when an input power is decreased. The value of gain also increases with the pump power [14]. Novak Stephanie et al [22] developed an analytic model for gain modulation in EDFAs. They considered small modulation index and computed gain according to different parameters. Modulation index is the ratio of over modulation amplitude and mean pump level. An erbium doped amplifier can amplify light wavelength ranging [24] from $1500 \mathrm{~nm}$ to more than $1600 \mathrm{~nm}$. Two such bands are in use today. One is the C-band (Conventional band) which occupies the spectrum from $1530 \mathrm{~nm}$ to $1560 \mathrm{~nm}$ and the second is L-band (Long wavelength band) which occupies the spectrum ranging from $1560 \mathrm{~nm}$ to $1610 \mathrm{~nm}$. Most EDFA works in the C-band. Yeh Chein-Chung et al [16] developed an s-band (1480-1520 nm) gain clamped EDFA by using optical feedback method. Noise is the second most important characteristic of an optical amplifier. The optical noise in an EDFA is called as Amplified Spontaneous Emission (ASE).The noise performance of an optical amplifier is quantified through noise figure (Fn) parameter which is defined as [28]

Fn $=($ SNR $)$ in $/($ SNR $)$ out

Where (SNR) in is the input signal to noise ratio and the (SNR) out is the output signal to noise ratio. Noise figure in an ideal EDFA is $3 \mathrm{~dB}$, while practical amplifiers can have noise figure as large as $6-8 \mathrm{~dB}$. Noise Figure can also be given by [28]

Fn $=\left(1+\frac{2 \text { Pase }}{\text { hv } \Delta \text { Vsp }}\right) 1 / G$

Where Fn is the noise figure, Pase is the ASE noise power, $h$ denotes the planks constant, Vsp is the bandwidth of EDFA and $\mathrm{G}$ denotes gain. The two most important elements in order to produce high gain in L-band EDFA are the length of EDF, the pump power and the signal wavelength [31]. In this paper we have analyzed the effect of pump power and the input signal power.

This paper is organized into six sections. In section 2, Literature has been discussed, while section 3 presents the work methodology and the proposed work. Section 4, demonstrates the model Simulation details. Section 5 presents the results and discussions. Finally, the paper is concluded in section 6 and Section 7, the paper lists all the references in section8.

\section{LITERATURE REVIEW}

EDFAs invention in late 80's has been a great achievement as it has given a new life to optical fiber communication systems Akhter Fowzia et al [1] Modeled and characterized all possible triple pass EDFA configurations. They determined optimum length and pump power ratio for each configuration and concluded that triple pass EDFA is the best for practical 
design. Chaugule Sachin et al [6] simulated WDM and Optical Amplifier. They recommended EDFA as a better option for optical amplifier due to its high gain and low noise. Naji W.A et al [7] proposed a new simulator which is capable of changing design parameters such as length, pump power and studies their effects on performance parameters. This paper [8] presented a quick review to the basic optical amplifier as technology and explains various configurations and models. Awaji Yoshinari et al [11] demonstrated various challenges towards burst mode EDFA.They optimized EDFA to suppress the transience intrinsically without any controlling such as AGC or Optical Feedback loop. Adolph Tamer et al [14] studied different features of the amplifier depending upon opto-geometric parameters and simulation results shows gain, power dependency on internal parameters.Tae Joon et al [17] proposed an All-Optical gain clamped Erbium-Doped Fiber Amplifier with improved noise Figure. It was based on reflecting amplified spontaneous emission (ASE) into EDFA.They experimentally demonstrated the scheme by using a coarse WDM Coupler at input fiber.Harun S.W et al[18] demonstrated a gain clamped L-Band EDFA with improved gain by incorporation of a broadband fiber brag grating(FBG).The Fiber Bragg Grating is a device which attenuates the backward propagating ASE.Subramaniam.T et al [19] presented the evaluation of design software capability in simulating any EDFA design and comparing the results with the experimental values.Izyani M.A[21] implemented a counter-propagating ring-laser cavity technique for gain clamped and flattened EDFA.They also compared the Gain and noise figure for with feedback and without feedback EDFA architectures. Hwang Seongtaek et al [24] demonstrated a broad-band EDFA with Double pass Configuration. They configured first stage for C-band and Lband amplification, while the second stage only amplifies the L-band signals.Kozak M.M [25] described EDFA_design and VPI Component Maker programs. A survey was made on commercially available software For EDFA design, Simulation and Optimization. It was concluded that such programs gave accurate results.Horiguchi Masaharu et al[30] suggested two different pumping wavelengths as $660 \mathrm{~nm}$ and $820 \mathrm{~nm}$ for the development of an efficient EDFA.The model with these pumping wavelengths experienced high gain and low noise Figure.

\section{WORK METHODOLOGY}

In this work, we proposed the simulation models of EDFA using Single and Multiple wavelength sources with Codirectional Pumping (980nm) scheme. The Pump power variation of $43 \mathrm{~mW}, 65 \mathrm{~mW}$ and $80 \mathrm{~mW}$ along with signal power ranging from $-30 \mathrm{dBm},-20 \mathrm{dBm},-10 \mathrm{dBm}$ and $0 \mathrm{dBm}$ have been simulated with WDM blocks and Isolators. We have presented a high Performance approach here that has not been used in this manner before for such design.

\subsection{Applied Methodology}

The applied methodology is based on Co-Propagating Pumping approach. All the blocks in the architecture have been tested and assembled together to compose the complete system. The resulting proposed model was simulated and the parameter values obtained were tabulated. For the present work, we have used an efficient EDFA design software tool to study simulation of basic EDFA model.Subramaniam. T et al [19] also simulated an EDFA system using EDFA_Design Software. The EDFA Design Software tool derives values for such as Gain, Noise Figure and ASE.Simulated results obtained are dependent on the options chosen and the parameters values set.

\subsection{Proposed Work}

Fig. 4 shows the proposed model for simulation of EDFA with Pumping wavelength of $980 \mathrm{~nm}$ using Co-directional (Forward) Pumping Scheme. The proposed model consist of either the single input Source (1550nm) or the Multiple Wavelength source with different channels (1520nm-1610nm) whose output is given to the isolator. An isolator [31] is a device which allows the propagation of light in only one direction with zero reflections. By using Co-directional pumping scheme, the output of an isolator is combined with the pump signal in a WDM Coupler. Wavelength Division Multiplexing (WDM) coupler used is the one that combines the input signal and the pump signal and it passes this combined signal to the EDFA.Ismail.N et al [26] presented 980nm pumped power variation effect on a Full Duplex Single EDF Bi-Directional Erbium Doped Fiber Amplifier. The proposed model consist of either the single input Source (1550nm) or the Multiple Wavelength source with different channels $(1520 \mathrm{~nm}-1610 \mathrm{~nm})$ whose output is given to the isolator.

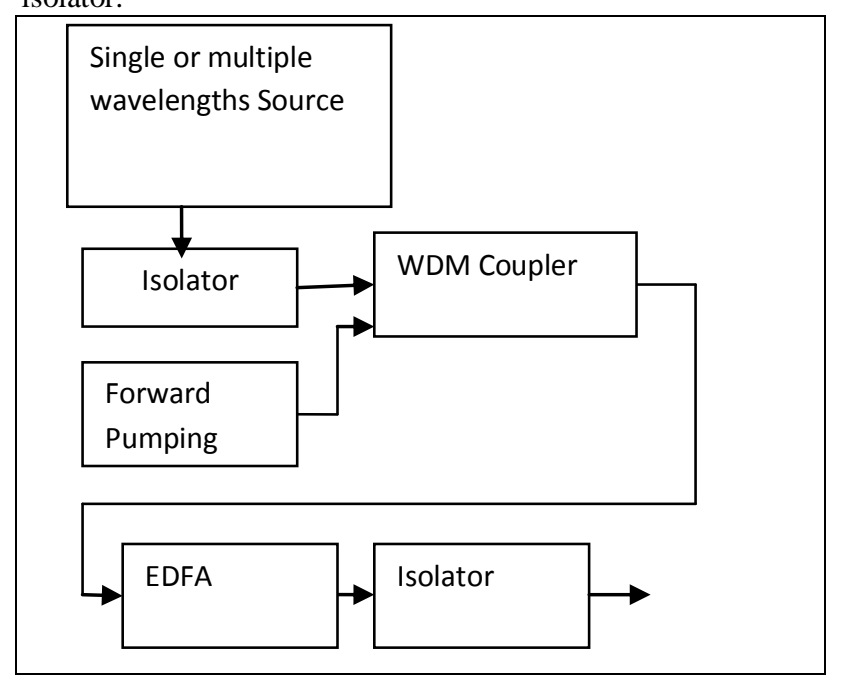

Fig. 4: Block Diagram of Single /Multiple wavelength sources with Co directional Pumping Scheme

An isolator [31] is a device which allows the propagation of light in only one direction with zero reflections. By using Codirectional pumping scheme, the output of an isolator is combined with the pump signal in a WDM Coupler. Wavelength Division Multiplexing (WDM) coupler used is the one that combines the input signal and the pump signal and it passes this combined signal to the EDFA_design software utilizes this model with all the components shown in the blocks. Parameters for the pump source (here used as $980 \mathrm{~nm}$ ), input signal source (single/multi wavelength) are varied so as to obtain optimized results. Graphs can be seen in the software itself for each changed value of parameters such as pump power and input signal power. The observations for required parameters such as gain, Noise figure and ASE can be carried out. Probe is used here between input and output to check overall gain and noise figure of the EDFA system. 


\section{PROPOSED MODEL SIMULATION}

The proposed EDFA model with single wavelength source $(1550 \mathrm{~nm})$ using Co-directional Pumping has been shown in the Fig.5.The wavelength for the pump source used is $980 \mathrm{~nm}$. The parameters Gain and Noise figure been measured with different pump powers $43 \mathrm{~mW}, 65 \mathrm{~mW}$ and $80 \mathrm{~mW}$ and with EDF length of $10 \mathrm{~m}$. Firstly, the pump power is kept constant and input signal power is varied and secondly the input power is kept constant and Pump power is varied.Semmalar.S et al [2] simulated EDFA of different lengths with an influence of pump power for optimizing gain and noise Figure.

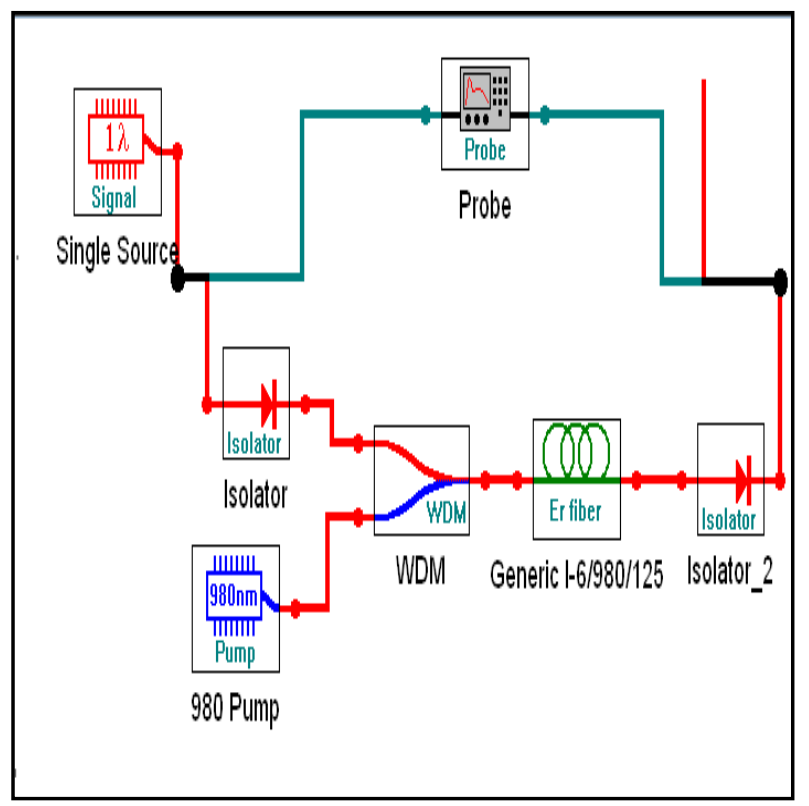

Fig.5: Simulated model of single wavelength source $(1550 \mathrm{~nm})$ with co-directional pumping scheme.

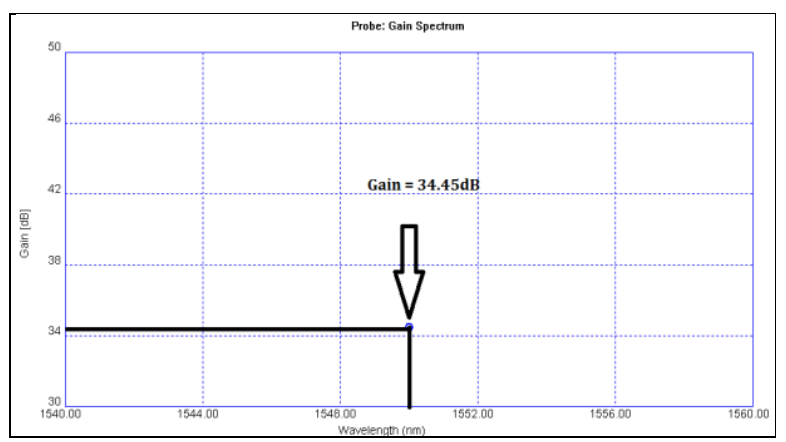

Fig.6.Simulated Gain spectrum for Single channel EDFA of length $=10 \mathrm{~m}$ with Input Signal Power $=-30 \mathrm{~dB}$ and Pumping power $=80 \mathrm{~mW}$ for Co-directional pumping.

The simulator was used for the implementation of single stage Erbium Doped Fiber Amplifier (EDFA) such as single pass (SP), double pass (DP) and triple-pass EDFA. Below Fig.6 is showing the Gain spectrum of single channel source EDFA of wavelength $=1550 \mathrm{~nm}$ with input signal power of $-30 \mathrm{~dB}$. Pumping source used here is $980 \mathrm{~nm}$, Insertion loss for isolator is $0.3 \mathrm{~dB}$ and the gain measured is $34.450 \mathrm{~dB}$. Fig.7 is showing the Noise Figure spectrum of simulated model with single wavelength source. Parameters like pump power and the input signal power [31] have an influence on the gain spectrum and as well as the noise Figure spectrum.

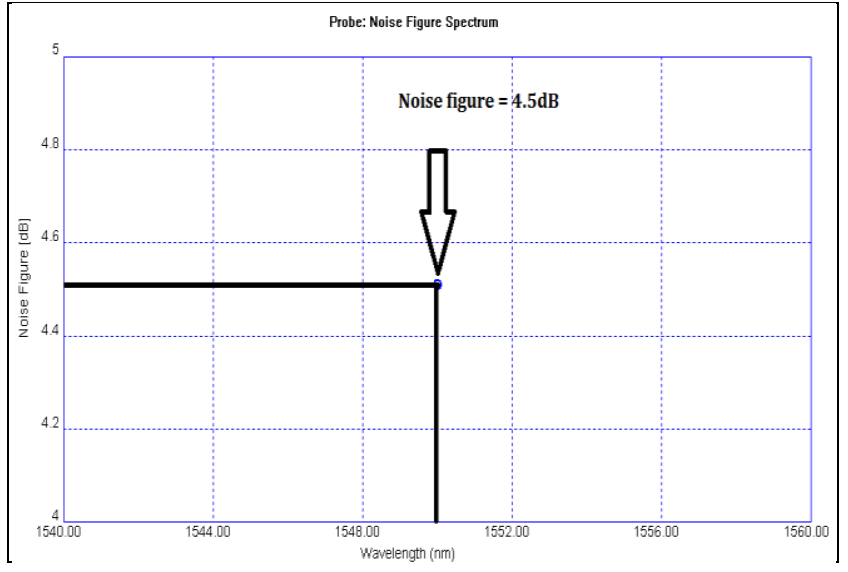

Fig.7: Simulated Noise spectrum for EDFA of length=10m with pumping power $=80 \mathrm{~mW}$ for Co-directional pumping Scheme.

The gain spectrum and the noise figure spectrum of EDFAs can vary from amplifier to amplifier even with the same core composition. The reason for this variation is dependency of gain spectrum on length of EDFA [31].Fig.8 is showing the simulated model of Multi-wavelength source over the wavelength ranging from $(1520 \mathrm{~nm}-1610 \mathrm{~nm})$ including Cband and L-band. Optimized Pump power used here is $65 \mathrm{mWand}$ Input Signal power is $-30 \mathrm{dBm}$. Insertion loss for isolator is $0.3 \mathrm{~dB}$. The maximum gain measured is $31.646 \mathrm{~dB}$ and the Noise Figure measured is 4.0dB. S.Semmalar [3] simulated an EDFA with optimized gain using Tri-Counter Directional Pumping scheme. It was justified that gain increases with increasing the pumping stages.

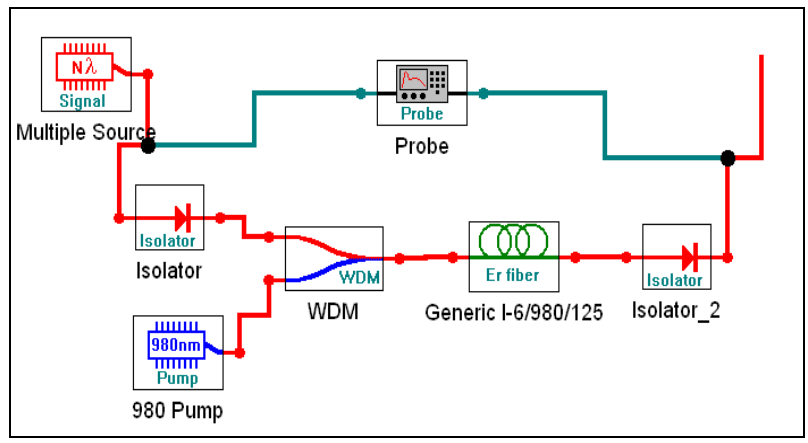

Fig.8: Simulated model of Multi- wavelength source with co-directional pumping scheme

Fig.9 is showing the gain spectrum for multi-channel EDFA with length $=10 \mathrm{~m}$. The absorption and emission cross sections having different spectral characteristics affect the gain spectrum [27]. Novak Stephanie [23] developed a simulink model using MATLAB for EDFA dynamics applied to gain modulation. This Simulink model used two wavelengths, one or the pump and another for the signal. 


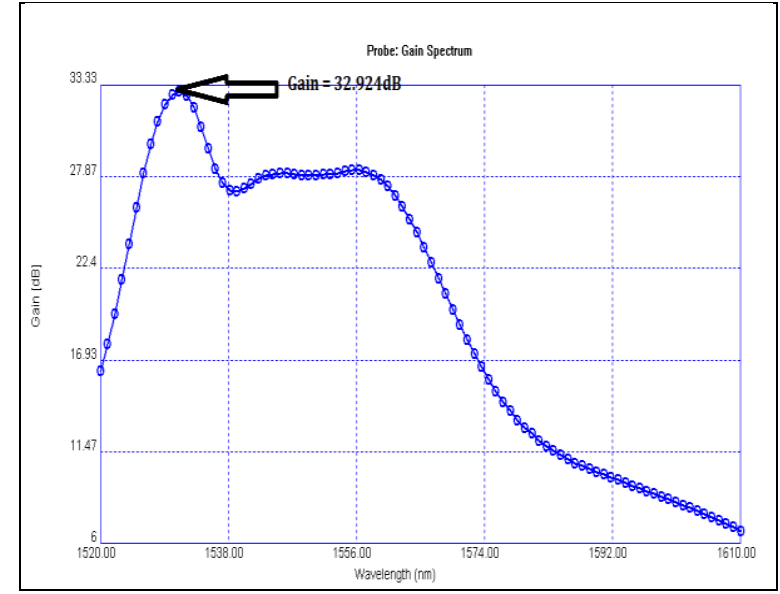

Fig. 9: Simulated Gain spectrum for Multi-channel EDFA of length $=10 \mathrm{~m}$ with Input Signal Power $=-30 \mathrm{~dB}$ and Pumping power $=80 \mathrm{~mW}$ for Co-directional pumping.

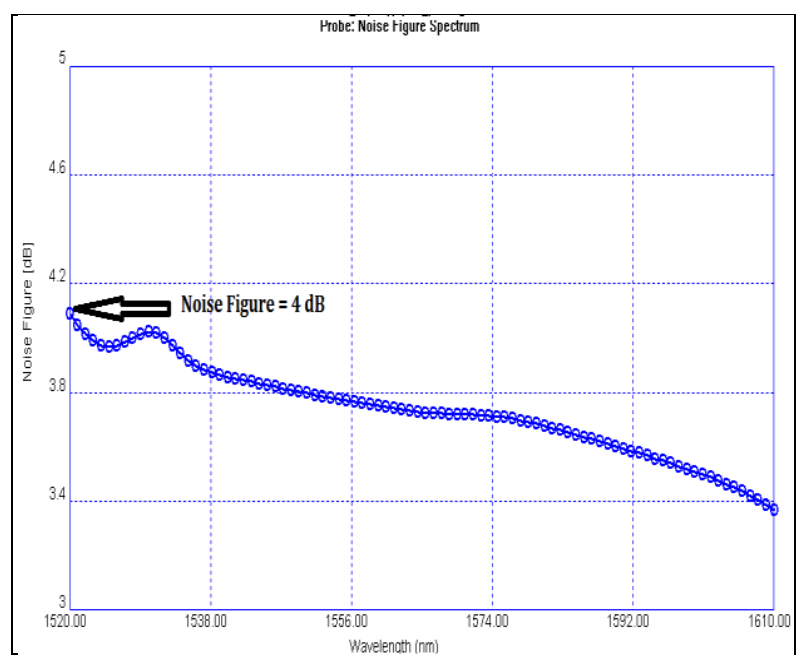

Fig.10: Simulated Noise spectrum for EDFA of length $=10 \mathrm{~m}$ with pumping power $=80 \mathrm{~mW}$ for Codirectional pumping.

Fig.10 is showing the noise spectrum for EDFA of length $10 \mathrm{~m}$ with pumping power of $80 \mathrm{~mW}$ using Co-directional Pumping Scheme.WDM technology will play a dominant role for the development of the next generation high-speed networks [5].

\section{RESULTS \& DISCUSSIONS}

The proposed model simulation results for both gain and noise figure shows change in their peak values. The simulation results have been shown for $(1550 \mathrm{~nm})$ for single wavelength source and for (1520nm-1610nm) for multi-wavelength source. The plotted values are tabulated with different pump power and input signal power in Table 1.

Simulation Results indicate that Gain and noise figures are affected by pump power and the Input Signal power variations.ASE is the Amplified Spontaneous which also varies with the changed parameters as indicated by the present results. Here we have observed only backward ASE.
TABLE 1 Results of comparison of Gain, Noise figure and ASE for different pump power and EDFA length $=10 \mathrm{~m}$ with forward Pumping scheme.

\begin{tabular}{|c|c|c|c|c|}
\hline \multirow{4}{*}{$\begin{array}{c}\text { Pump } \\
\text { Power }\end{array}$} & $\begin{array}{c}\text { Signal } \\
\text { Input } \\
\text { power } \\
(\mathbf{d B m})\end{array}$ & $\begin{array}{c}\text { Gain(dB } \\
\text { ) }\end{array}$ & $\begin{array}{c}\text { NF(dB } \\
\text { ) }\end{array}$ & $\begin{array}{c}\text { Backwar } \\
\text { d } \\
\text { SE(dBm) }\end{array}$ \\
\hline \multirow{4}{*}{$43 \mathrm{~mW}$} & -30 & 32.193 & 4.5 & 0.16 \\
\cline { 2 - 5 } & -20 & 29.765 & 4.2 & -2 \\
\cline { 2 - 5 } & -10 & 22.649 & 3.8 & -10 \\
\cline { 2 - 5 } & 0 & 13.351 & 4.41 & -23 \\
\hline \multirow{4}{*}{$65 \mathrm{~mW}$} & -30 & 33.73 & 4.5 & 2.5 \\
\cline { 2 - 5 } & -20 & 31.537 & 4.2 & 0.333 \\
\cline { 2 - 5 } & -10 & 24.58 & 3.7 & -8 \\
\cline { 2 - 5 } & 0 & 15.24 & 4.1 & -20 \\
\hline \multirow{4}{*}{$80 \mathrm{~mW}$} & -30 & 34.45 & 4.5 & 4 \\
\cline { 2 - 5 } & -20 & 32.378 & 4.2 & 1.429 \\
\cline { 2 - 5 } & -10 & 25.532 & 3.7 & -8 \\
\cline { 2 - 5 } & 0 & 16.175 & 4.1 & -20 \\
\hline
\end{tabular}

Table 1 shows the Results of comparison of Gain, ASE and noise figure for different pump power and EDFA length $=10 \mathrm{~m}$ with Co-directional Pumping scheme.

Following points can be summarized from the results shown in the table for Single wavelength source:

i. Keeping the pump power constant when signal power is decreased there is an increase in gain, $0.2 \mathrm{db}$ change in N.F and ASE power gets increased.

ii. Proposed results clearly show that pump power is proportional to gain as well as ASE. By fair choice of the signal input power and pump power we can optimize Gain, N.F, ASE.

iii. $\quad 80 \mathrm{mw}$ pump power with signal energy $=-10 \mathrm{dBm}$ shows lowest N.F of $3.7 \mathrm{~dB}$ with average gain $=25.532 \mathrm{~dB}$. Also compared to other values ASE is minimized so, it can be considered as an Optimized value.

iv. Lowest value of ASE is accounted for increased signal power and that can also be decreased by decreasing pump power.

As we see in the proposed results Pump power has a significant effect on both gain and noise figure. The direction of pump also affects the value of gain and noise figure.

Table 2 results shows that for a multi-wavelength source the optimized values can be taken at Pump Power $80 \mathrm{~mW}$ and Input Signal Power of $-30 \mathrm{dBm}$ where the gain comes out to be maximum that is $32.924 \mathrm{~dB}$ and the Noise Figure is minimum that is $4 \mathrm{~dB}$. 
TABLE 2 Results of comparison of Gain, ASE and noise figure for different pump power and EDFA length $10 \mathrm{~m}$ with Forward Pumping scheme

\begin{tabular}{|c|c|c|c|c|}
\hline \multirow{4}{*}{ Pump Power } & $\begin{array}{c}\text { Signal } \\
\text { Input } \\
\text { power } \\
\text { (dBm) }\end{array}$ & Gain(dB) & NF(dB) & $\begin{array}{c}\text { Backward } \\
\text { ASE(dBm) }\end{array}$ \\
\hline \multirow{4}{*}{$43 \mathrm{~mW}$} & -30 & 28.947 & 4.3 & -8 \\
\cline { 2 - 5 } & -20 & 18.116 & 5.8 & -19 \\
\cline { 2 - 5 } & -10 & 8.589 & 12.06 & -30 \\
\cline { 2 - 5 } & 0 & 2.489 & 23 & -37 \\
\hline \multirow{5}{*}{$65 \mathrm{~mW}$} & -30 & 31.646 & 4 & -5 \\
\cline { 2 - 5 } & -20 & 19.91 & 5.1 & -16 \\
\cline { 2 - 5 } & -10 & 10.5 & 11 & -29 \\
\cline { 2 - 5 } & 0 & 2.98 & 23 & -36 \\
\hline \multirow{5}{*}{$80 \mathrm{~mW}$} & -30 & 32.924 & 4 & -4 \\
\cline { 2 - 5 } & -20 & 20.761 & 4.9 & -15 \\
\cline { 2 - 5 } & -10 & 11.457 & 10 & -28 \\
\cline { 2 - 5 } & 0 & 3.3767 & 21 & -35 \\
\hline
\end{tabular}

Following points can be summarized from the above results for Multi-wavelength source:

i. Gain of $32.924 \mathrm{db}$ is obtained for a higher pump power without changing any other parameters shows an efficient technique.

ii. Here both L,C -Bands are used as source wavelength Other than these wavelengths ,for any desired window results shows improvement over gain and noise figure as with limited range stimulated emission show better progression.

iii. To reduce ASE we can vary signal power down below to $0 \mathrm{db}$ without changing pump power.

iv. $\quad-30 \mathrm{dBm}$ signal power value for any pump power shows maximum values of gain and comparatively the lowest values of noise figure.

J.A Carmelo et al [15] proposed the pump direction influence on EDFA. They used both co-propagating and counterpropagating schemes with optical feedback. The pump power values used for co-propagating scheme were $43 \mathrm{mw}, 65 \mathrm{mw}$ and $75 \mathrm{mw}$.The input signal power used was $-30 \mathrm{dBm}$. They considered feedback attenuator losses in the range 0 to $50 \mathrm{~dB}$. The gains and noise figure obtained were $28 \mathrm{~dB}$ and $7 \mathrm{~dB}$.

The values of pump power used in our proposed work are $43 \mathrm{mw}, 65 \mathrm{mw}$ and $80 \mathrm{mw}$ which are similar as used by J.A Carmelo [15].The simulation results obtained shows that the gain obtained at $-30 \mathrm{dBm}$ input power for single wavelength source is $34.45 \mathrm{~dB}$ and for multi-wavelength source is $32.924 \mathrm{~dB}$. The values obtained for noise figure are $4.5 \mathrm{~dB}$ for single wavelength source and $4 \mathrm{~dB}$ for multi-wavelength source. If our proposed results are compared with the paper [15], we find that our results are better in terms of gain and noise figure.

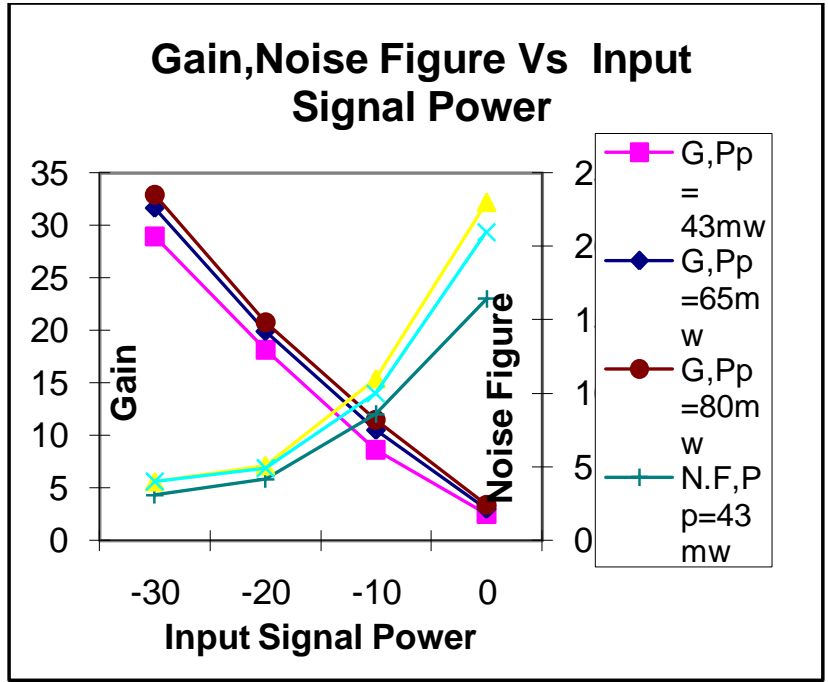

Fig.11: Gain and Noise Figure as a Function of Input Signal Power for single wavelength source usiing codirectional Pumping

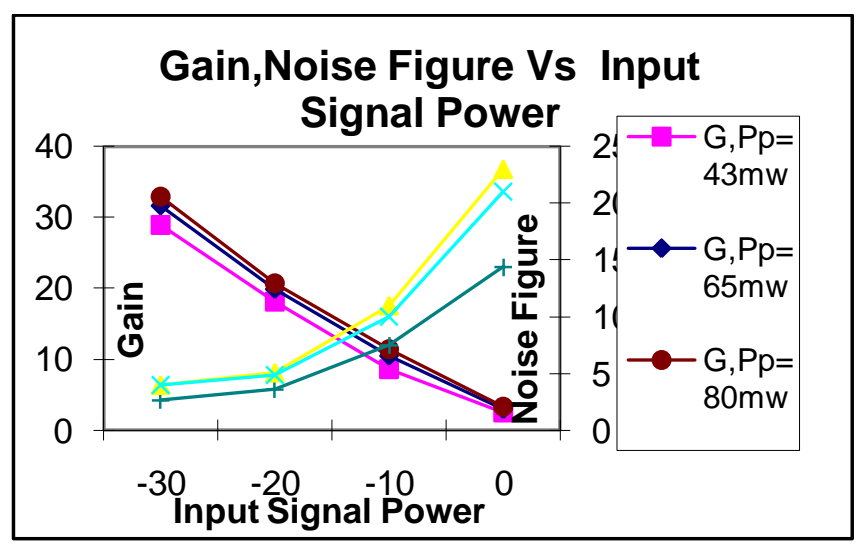

Fig.12: Gain and Noise Figure as a Function of Input

Signal Power for Multiwavelength source using Codirectional Pumping.

Fig $11 \& 12$ are showing the comparison graphs for gain and noise figure using single and multiple wavelength sources. Result observations show a mapping between pump power and input signal power over noise figure, ASE and gain. If any of the parameter has a limitation to be changed, other can be utilized to perform the same operation. Comparison graphs show that gain increases with increase in pump power. The reason is that gain depends on both the absorption and emission cross sections [27].

\section{CONCLUSION AND FUTURE ASPECTS}

We have proposed and simulated the EDFA model with single and multi-wavelength sources using pumping source of 980 $\mathrm{nm}$ wavelength. The various results were also compared. It is important to understand the desired range of wavelength used in EDFA which provides efficient results. Along with source wavelength if other parameters like length, pump power, signal power are changed, than optimized values of gain and noise figure are obtained. Thus, we have shown that the proposed model of an EDFA utilizing both single and multiwavelength sources was successfully simulated using WDM. 
For each pump power signal power is changed and we observed the changes in gain and noise figure. We have also shown the impact of change on ASE i.e. the major noise component in EDFA systems. Any of the desired condition of maximum gain and low noise or minimum ASE can be achieved using the values shown in the proposed results and also without changing each and every component. It may be observed that the Gain is optimized and Noise Figure initially decreases with increase in Pump Power and then attains the same value.

In future work, the model can be modified in different ways. Firstly, we can take this model and go with another parameter i.e. length of fiber and the Concentration of erbium ions using $1480 \mathrm{~nm}$ pumping. Secondly, cascaded model employing two EDFA's can be used with same parameters to have better results. Thirdly with the addition of new wavelengths the proposed model can be used network re-configuration. And the last is optical feedback method and circulators can be deployed on proposed model.

\section{ACKNOWLEDGMENTS}

We would like to thank Prof Rajesh Kumar, for his encouragement and support to develop this work.

\section{REFERENCES}

[1] Akhter Fowzia et al, "Modeling and characterization of all possible triple pass EDFA configurations", International Journal of Physical Sciences Vol.7 (18), pp.2656-2663, May 9, 2012

[2] Semmalar S., Poonkuzhali, Devi.P, "Optimized Gain EDFA of different lengths with an influence of pump power", IEEE Paper, 2011

[3] Semmalar S., Gujral Jyoti, "EDFA with Optimized Gain using Tri-Counter Directional Pumping", International Journal of Applied Engineering Research, ISSN 0973- 4562,Vol.6 No.5,April 2011.

[4] Naji W.A et al, "Review of Erbium-doped fiber amplifier", International Journal of Physical Sciences Vol.6(20), pp 4674-4689,September 23, 2011.

[5] Goel Aditya et al, "Design of broadband EDFA for next generation optical networks", journal of Neural Networks and applications, pp.9-13 Jan - June 2011.

[6] Chaugule Sachin et al, "WDM and Optical Amplifier", 2nd International Conference on Mechanical ans Electronics Engineering (ICMEE 2010) IEEE Paper 2010

[7] Naji W.A et al, "A Computer based Simulator for Erbium-Doped Fiber Amplifier", May 11-13, IEEE Paper 2010

[8] Introduction to optical amplifiers (white paper) Jun 2010.

[9] Berkdemir Cuneyt et al, "On the TemperatureDependent Gain and Noise Figure Analysis of CBand High- Concentration EDFAs with te effect of Cooperative Upconversion”, IEEE Journal of Lightwave Technology, Vol.27, No.9 May 2009

[10] Introduction to EDFA technology (white paper) June 2009.

[11] Awaji Yoshinari et al, "Challenges towards BurstMode EDFA”, IEEE Paper 2009
[12] Martini M.M.J et al, "Multi-pump Optimization for Raman + EDFA Hybrid Amplifiers under Pump Residual Recycling", IEEE Paper 2009

[13] Bakar Abu Hafiz Muhammad et al, "L-band Erbium- doped Fiber Amplier Pumped by 1455nm Laser Source for Repeaterless Transmission Systems ”, IEEE Paper 2008

[14] Tamer Adolph, Ould Saadi H. and Boutaleb A., "Simulation based analysis of erbium doped fiber amplifier (EDFA)", Journal of applied science, ISSN 1812-5654, Asian network for scientific information 2006

[15] Bastos-Fillho J.A Carmelo et al, "Influence of Pump Direction in All-Optical GainClamped Erbium Doped Fiber Amplifier", IEEE Paper 2005

[16] Yeh Hung-Chien et al, "S Band Gain-Clamped Erbium- Doped Fiber Amplifier by Using Optical Feedback Method", IEEE Photonics Technology Letters, VOL.16, No.1, Jan 2004

[17] Tae Joon et al, "All-Optical Gain-Clamped ErbiumDoped Fiber Amplifier with Improved Noise Figure and Freedom from Relaxation Oscillation", IEEE Photonics Technology Letters, VOL.16, No.1, Jan 2004

[18] Harun S.W et al, "Gain Clamping in Two-Stage LBand EDFA Using a Broadband FBG", IEEE Photonics VOL.16,No.2,February 2004 Technology Letters,

[19] Subramanian T., Harun S.W., Mahdi M.A., Poopalan P. \& Ahmad H., "simulation of EDFA system using EDFA_design software", Urnal Technology, 40(D) June2004 University Technology Malaysia

[20] Choi Bo-Hun et al, "New Pump Wavelength of 1540- $\quad \mathrm{nm}$ Band for Long-Wavelength-Band Erbium Doped Fiber Amplifier", IEEE Journal of Quantum Technology, Vol 39,No.10 October 2003

[21] Izyani M.A Mas et al, "Flattened Dual-Stage GCEDFA Using Fiber - Ring Laser Technique", IEEE Paper 2003

[22] Novak Stephanie et al, "Analytic model for gain modulation in EDFA", IEEE Journal of Light wave Technology, vol.20, No.6, June 2002.

[23] Novak Stephanie et al, "Simulink Model for EDFA Dynamics Applied to Gain Modulation", IEEE Journal of Light wave Technology, vol.20, No.6, June 2002.

[24] Hwang Seongtaek et al, "Broad-Band ErbiumDoped Fiber Amplifer with Double-Pass Configuration", IEEE Photonics Technology Letters, vol.13, No.12, December 2001

[25] Kozak M.M and Caspary R., "Computer Aided EDFA Design, Simulation and Optimization”, IEEE Paper, 2001

[26] Ismali.N, "980NM-Pumped Power Variation Effect on a Full Duplex Single EDF Bi-Directional Erbium Doped Fiber Amplifier”, IEEE Paper, 2000

[27] Aggarwal G.P, Non Linear Fiber Optics, 3rd edition, Academic Press, New York, 2001

[28] Becker P. C., Olsson N. A., Simpson J. R. ErbiumDoped Fiber Amplifiers Fundamentals and Technology, Academic Press, San Diego, 1999. 
[29] Kobayashi M, "Noise Figure improvement of optical

gain clamped fiber amplifier by mid-point band reject filter for lasing light", Electronics Letter,vol.35,No.6, March 1999.

[30] Horiguchi Masaharu, Erbium Doped Fiber Amplifiers

Pumped in 660nm and $820 \mathrm{~nm}$ bands IEEE Journal of Light wave technology, Vol 12, no.5, May 1994.

[31] Mynbaev D.K, Scheiner L.L, "Fiber-Optic Communications Technology", Pearson Education, Inc. and Dorling Kindersley Publishing Inc., 2001 